\title{
Confluent Expansions for Functions of Two Variables
}

\author{
By V. L. Deshpande
}

\begin{abstract}
In a recent paper, J. L. Fields established four theorems giving confluent expansions for functions of one variable. In the present paper, we extended one theorem of Fields for functions of two variables. The usefulness of the theorem is illustrated by obtaining known and hitherto unknown transformations for Appell functions and Horn functions.
\end{abstract}

1. This paper is a continuation of an earlier work of the author's (see [1], [2] and [3]). It discusses the extension of some results of Fields [4] to functions of two variables.

The usefulness of Theorem 1 below is illustrated by obtaining known and hitherto unknown transformations for functions of two variables such as Appell functions $F_{1}, F_{2}$ and Horn functions $H_{2}$ and $H_{4}$ [5, pp. 224-225, (6), (7), (14) and (16)]. The Horn function $\mathrm{H}_{4}$ is expressed as a generating function for the Jacobi and Legendre polynomials.

With the help of the theorem, an asymptotic confluent expansion for the Kampé de Fériet function [6, p. 150] is established.

2. THEOREM 1. If

$$
V(x, y)=\sum_{m, n=0}^{\infty} A_{m n}(x)^{m}(x y)^{n}, \quad|x|<r,|x y|<s,
$$

then the series

$$
S(x, x y, \sigma)=\sum_{m, n=0}^{\infty} A_{m n} \frac{(\sigma+a)_{m+n}\left(\sigma+a^{\prime}\right)_{n}}{(\sigma+b)_{m+n}\left(\sigma+b^{\prime}\right)_{n}}(x)^{m} \cdot(x y)^{n}
$$

is convergent for $|x|<r,|x y|<s, \sigma+b \neq 0,-1,-2, \cdots,\left(\sigma+b^{\prime}\right) \neq 0,-1$, $-2, \cdots$, and can be rearranged in the region $|x|<r / 2,|x y|<s / 2$ as

$$
S(x, x y, \sigma)=\sum_{i, k=0}^{\infty} g_{i, k}(x, y)\left[(\sigma+b)_{j}\left(\sigma+b^{\prime}\right)_{k}\right]^{-1},
$$

where $g_{i, k}(x, y)$ is defined by (2.8) below. Also, if $a, a^{\prime}, b, b^{\prime}$ are bounded, then we obtain the asymptotic confluent expansion of $S(x, x y, \sigma)$ as

$$
\begin{aligned}
& S(x, x y, \sigma) \sim \sum_{i, k=0}^{\infty} g_{i, k}(x, y)\left[(\sigma+b)_{j}\left(\sigma+b^{\prime}\right)_{k}\right]^{-1}, \quad \sigma \rightarrow \infty \\
& |\arg (\sigma+b)| \leqq \pi-\delta,\left|\arg \left(\sigma+b^{\prime}\right)\right| \leqq \pi-\delta^{\prime}, \delta, \delta^{\prime}>0,|x|<r,|x y|<s .
\end{aligned}
$$

Received March 22, 1973.

AMS (MOS) subject classifications (1970). Primary 33A30.

Copyright (c) 1974, American Mathematical Society 
Furthermore, (2.4) can be expressed in negative powers of $\sigma$ if we employ the formula in [7] for $\left[(\sigma+b)_{i}\left(\sigma+b^{\prime}\right)_{k}\right]^{-1}$.

Proof. From (2.1) and the test of convergence given by Horn [5, p. 227], it can be proved that (2.2) is convergent for $|x|<r,|x y|<s$.

From [8, p. 69, Example 4],

$$
\begin{aligned}
\frac{(\sigma+a)_{m+n}}{(\sigma+b)_{m+n}} & =\sum_{j=0}^{\infty} \frac{(b-a)_{j}(-m-n)_{i}}{(\sigma+b)_{i} j !}, \quad \sigma+b \neq 0,-1,-2, \cdots, \\
\frac{\left(\sigma+a^{\prime}\right)_{n}}{\left(\sigma+b^{\prime}\right)_{n}} & =\sum_{k=0}^{\infty} \frac{\left(b^{\prime}-a^{\prime}\right)_{k}(-n)_{k}}{k !\left(\sigma+b^{\prime}\right)_{k}}, \quad \sigma+b^{\prime} \neq 0,-1,-2, \cdots,
\end{aligned}
$$

and

$$
\frac{\partial^{j+k} V(x, y)}{\partial x^{j} \cdot \partial y^{k}}=\sum_{m, n=0}^{\infty} A_{m n} \cdot(-m-n)_{j}(-n)_{k}(-1)^{i+k} \cdot x^{m+n-j} \cdot y^{n-k} .
$$

Using (2.5)-(2.7) in (2.2) and comparing with (2.3), we obtain

$$
g_{i, k}(x, y)=\frac{(b-a)_{i}\left(b^{\prime}-a^{\prime}\right)_{k}(-x)^{i} \cdot(-y)^{k}}{j ! k !} \frac{\partial^{i+k}}{\partial x^{i} \cdot \partial y^{k}} V(x, y) .
$$

Now we proceed to obtain convergence conditions for (2.3).

Let $|x|<x_{0}<r$ and $|x y|<x_{0} \cdot y_{0}<s$. Then

$$
A_{m, n}=o\left[\left(x_{0}\right)^{-m-n} \cdot\left(y_{0}\right)^{-n}\right] \text {. }
$$

From (2.3),

$$
\begin{aligned}
|S(x, x y, \sigma)| & \leqq \sum_{i, k=0}^{\infty}\left|\frac{(b-a)_{j}\left(b^{\prime}-a^{\prime}\right)_{k}}{(\sigma+b)_{j}\left(\sigma+b^{\prime}\right)_{k}}\right| \frac{|x|^{i} \cdot|y|^{k}}{j ! k !}\left|\frac{\partial^{j+k} V(x, y)}{\partial x^{j} \cdot \partial y^{k}}\right| \\
& =\sum_{i, k=0}^{\infty}\left|\frac{(b-a)_{i}\left(b^{\prime}-a^{\prime}\right)_{k}}{(\sigma+b)_{j}\left(\sigma+b^{\prime}\right)_{k}}\right| \cdot \frac{|x|^{i} \cdot|y|^{k}}{j ! k !} \frac{\partial^{j+k}}{\partial x^{j} \cdot \partial y^{k}} \sum_{m, n=0}^{\infty}\left|\frac{x}{x_{0}}\right|^{m} \cdot\left|\frac{x y}{x_{0} y_{0}}\right|^{n} \\
& =\sum_{m, n=0}^{\infty} \sum_{i=0}^{m+n} \sum_{k=0}^{n}\left|\frac{(b-a)_{i}\left(b^{\prime}-a^{\prime}\right)_{k}}{(\sigma+b)_{j}\left(\sigma+b^{\prime}\right)_{k}}\right| \cdot \frac{(-m-n)_{j}(-n)_{k}}{j ! k !}\left|\frac{x}{x_{0}}\right|^{m} \cdot\left|\frac{y}{y_{0}}\right|^{n} .
\end{aligned}
$$

Employing formula [8, p. 57, (2)], we obtain after some simplification,

$$
\begin{aligned}
|S(x, x y, \sigma)| \leqq & \sum_{m, n, i, k=0}^{\infty}\left|\frac{(b-a)_{j}\left(b^{\prime}-a^{\prime}\right)_{k}}{(\sigma+b)_{j}\left(\sigma+b^{\prime}\right)_{k}}\right| \frac{(j+1)_{m+n} \cdot(k+1)_{n}}{(m+n) ! n !} \cdot\left|\frac{x}{x_{0}}\right|^{m+n+i} \cdot\left|\frac{x}{y_{0}}\right|^{n+k} \\
= & \left(1-\left|\frac{x}{x_{0}}\right|\right)^{-1} \cdot\left(1-\left|\frac{y}{y_{0}}\right|\right)^{-1} \cdot{ }_{2} F_{1}\left[\begin{array}{c}
\left.b-a, 1, \frac{1}{\left|\frac{x_{0}}{x}\right|-1}\right] \\
\sigma+b, \mid
\end{array}\right. \\
& \cdot{ }_{2} F_{1}\left[\begin{array}{c}
b^{\prime}-a^{\prime}, 1, \frac{1}{\sigma+b^{\prime}}\left|\frac{y_{0} \mid}{y}\right|-1
\end{array}\right]
\end{aligned}
$$

which is convergent for $|x|<r / 2,|x y|<s / 2$. Hence, (2.3) is proved.

We can easily show that the $g_{i, k}(x, y)$ in (2.4) are the Poincaré coefficients of $s(x, x y, \sigma)$ as $\sigma \rightarrow \infty$.

Special Cases. (i) Putting $a=b$ and $a^{\prime}=b^{\prime}$ in Theorem 1, we obtain the follow- 
ing theorems:

THEOREM 2. If

$$
V(x, y)=\sum_{m, n=0}^{\infty} A_{m n} \cdot(x)^{m} \cdot(x y)^{n}, \quad|x|<r,|x y|<s,
$$

and

$$
S(x, x y, \sigma)=\sum_{m, n=0}^{\infty} A_{m n} \frac{\left(\sigma+a^{\prime}\right)_{n}}{\left(\sigma+b^{\prime}\right)_{n}}(x)^{m} \cdot(x y)^{n}, \quad|x|<r,|x y|<s,
$$

then

$$
S(x, x y, \sigma)=\sum_{k=0}^{\infty} \frac{\left(b^{\prime}-a^{\prime}\right)_{k}(-y)^{k}}{k !\left(\sigma+b^{\prime}\right)_{k}} \cdot \frac{\partial^{k} V(x, y)}{\partial y^{k}}, \quad|x|<r / 2,|x y|<s / 2 .
$$

THEOREM 3. If

$$
V(x, y)=\sum_{m, n=0}^{\infty} A_{m n} \cdot(x)^{m} \cdot(x y)^{n}, \quad|x|<r,|x y|<s,
$$

then the series

$$
S(x, x y, \sigma)=\sum_{m, n=0}^{\infty} A_{m n} \frac{(\sigma+a)_{m+n}}{(\sigma+b)_{m+n}}(x)^{m}(x y)^{n}, \quad|x|<r,|x y|<s,
$$

can be rearranged to read

$$
S(x, x y, \sigma)=\sum_{j=0}^{\infty} \frac{(b-a)_{i} \cdot(-x)^{i}}{j !(\sigma+b)_{i}} \frac{\partial^{i} V(x, y)}{\partial x^{i}}, \quad|x|<r / 2,|x y|<s / 2 .
$$

3. In this section, we establish various transformation formulae and generating relations for functions of two variables. The result (3.6) is important as it contains all known transformations [5, p. 240, (6)-(8)]. Formula (3.7) is known [5, p. 239, (1)], but the technique for obtaining it is new. The rest of the formulae are believed to be new.

We establish the following results.

(a) $H_{4}\left(\alpha, \sigma+a^{\prime}, \gamma, \sigma+b^{\prime}, x, x y\right)$

$$
\begin{aligned}
& =(1-x y)^{-\alpha} \cdot H_{4}\left(\alpha, b^{\prime}-a^{\prime}, \gamma, \sigma+b^{\prime}, \frac{x}{(1-x y)^{2}}, \frac{x y}{x y-1}\right) \\
& =(1+2 \sqrt{ } x)^{-\alpha} \cdot F_{2}\left(\alpha, \sigma+a^{\prime}, \frac{2 \gamma-1}{2},\right. \\
& \left.\quad \sigma+b^{\prime}, 2 \gamma-1, \frac{x y}{1+2 \sqrt{ } x}, \frac{4 \sqrt{ } x}{1+2 \sqrt{ } x}\right), \\
& \text { (b) } x^{-\alpha} \cdot H_{4}\left(\alpha, \sigma+a^{\prime}, \frac{\alpha+1}{2}, \sigma+b^{\prime}, \frac{x^{2}-1}{4 x^{2}}, \frac{\left(x^{2}-1\right) y}{4 x^{2}}\right)
\end{aligned}
$$

$$
=\sum_{k=0}^{\infty} \frac{(\alpha)_{k}\left(\sigma+a^{\prime}\right)_{k}}{\left(\sigma+b^{\prime}\right)_{k}\left(\frac{\alpha+1}{2}\right)_{k}}\left[\frac{\left(x^{2}-1\right) y}{4 x}\right]^{k} \cdot P_{k}^{((\alpha-1) / 2,(\alpha-1) / 2)}(x)
$$


provided $\left|\left(x^{2}-1\right) / 4 x^{2}\right|<r,\left|\left(x^{2}-1\right) y / 4 x^{2}\right|<s$ and $4 r=(s-1)^{2}$.

$$
H_{4}\left(\alpha, \sigma+a^{\prime}, \gamma, \sigma+b^{\prime}, x, x y\right)
$$

$$
=x^{\alpha} \cdot F_{4}\left(\alpha, \sigma+a^{\prime}, \frac{\alpha+1}{2}, \frac{\alpha+1}{2}, \frac{1}{2} y(x-1), \frac{1}{2} y(x+1)\right) \text {, }
$$

(c) $H_{2}\left(\alpha, \sigma+a^{\prime}, \gamma, \delta, \sigma+b^{\prime}, x y, x\right)$

$$
=(1-x y)^{-\alpha} \cdot H_{2}\left(\alpha, b^{\prime}-a^{\prime}, \gamma, \delta, \sigma+b^{\prime}, \frac{x y}{x y-1}, x(1-x y)\right),
$$

(d) $F_{2}\left(\alpha, \beta, \sigma+a^{\prime}, \gamma, \sigma+b^{\prime}, x, x y\right)$

$$
=F_{A}\left(\alpha, \beta, \beta^{\prime}, b^{\prime}-a^{\prime}, \gamma, \beta^{\prime}, \sigma+b^{\prime}, x, x y,-x y\right),
$$

(e) $F_{1}\left(\sigma+a, \beta, \beta^{\prime}, \sigma+b, x, x y\right)$

$$
=(1-x)^{-\beta} \cdot(1-x y)^{-\beta^{\prime}} \cdot F_{1}\left(b-a, \beta, \beta^{\prime}, \sigma+b, \frac{x}{x-1}, \frac{x y}{x y-1}\right) .
$$

Proof. (a) Setting $A_{m n}=(\alpha)_{2 m+n} /(\gamma)_{m} m ! n$ ! in Theorem 2, we have, on simplification,

$$
\begin{aligned}
S(x, x y, \sigma) & =H_{4}\left(\alpha, \sigma+a^{\prime}, \gamma, \sigma+b^{\prime}, x, x y\right) \\
& =\sum_{m, n=0}^{\infty} \sum_{k=0}^{n} \frac{\left(b^{\prime}-a^{\prime}\right)_{k}(\alpha)_{2 m+n}(-n)_{k}(x)^{m}(x y)^{n}}{k ! m ! n !\left(\sigma+b^{\prime}\right)_{k}(\gamma)_{m}} \\
& =\sum_{m, n, k=0}^{\infty} \frac{(\alpha)_{2 m+n+k}\left(b^{\prime}-a^{\prime}\right)_{k}(x)^{m}(x y)^{n}(-x y)^{k}}{k ! m ! n !\left(\sigma+b^{\prime}\right)_{k}(\gamma)_{m}}
\end{aligned}
$$

in view of $[8$, p. $56,(1)]$.

In (3.8), summing the series over $n$, we immediately arrive at (3.1).

Again, in (3.8), summing the series over $m$ and employing formula [8, p. 22, Lemma 5], we obtain

$$
\begin{aligned}
& H_{4}\left(\alpha, \sigma+a^{\prime}, \gamma, \sigma+b^{\prime}, x, x y\right) \\
& \quad=\sum_{n, k=0}^{\infty} \frac{(\alpha)_{n+k}\left(b^{\prime}-a^{\prime}\right)_{k}(x y)^{n} \cdot(-x y)^{k}}{n ! k !\left(\sigma+b^{\prime}\right)_{k}} \cdot{ }_{2} F_{1}\left[\frac{\alpha+n+k}{2}, \frac{\alpha+n+k+1}{2}, 4 x\right] \\
& \quad=\sum_{k=0}^{\infty} \sum_{n=0}^{k} \frac{\left(b^{\prime}-a^{\prime}\right)_{k-n}(\alpha)_{k}(-1)^{k-n}(x y)^{k}}{n !(k-n) !\left(\sigma+b^{\prime}\right)_{k-n}}{ }_{2} F_{1}\left[\frac{\alpha+k}{2}, \frac{\alpha+k+1}{2}, 4 x\right]
\end{aligned}
$$

in view of $[8$, p. $56,(1)]$.

Reversing the order of the inner summation and summing the series over $n$, we arrive at

$$
\begin{aligned}
H_{4}(\alpha, \sigma & \left.+a^{\prime}, \gamma, \sigma+b^{\prime}, x, x y\right) \\
& =\sum_{k=0}^{\infty} \frac{(\alpha)_{k}\left(\sigma+a^{\prime}\right)_{k}}{k !\left(\sigma+b^{\prime}\right)_{k}}(x y)^{k} \cdot{ }_{2} F_{1}\left[\frac{\alpha+k}{2}, \frac{\alpha+k+1}{2}, 4 x\right] .
\end{aligned}
$$


In (3.9), employing formula [5, p. 112, (17)] and expressing the hypergeometric function in series form, we obtain the transformation formula (3.2).

(b) Again in (3.9), making use of formula [5, p. 112, (16)], substituting $2 \gamma=$ $\alpha+1$, replacing $x$ by $\left(x^{2}-1\right) / 4 x^{2}$ and employing formula [5, p. 254, (11)], we obtain a generating function for the Jacobi polynomial given by (3.3).

In (3.3), substituting $\sigma+b^{\prime}=(\alpha+1) / 2$ and replacing $y$ by $4 x y /\left(x^{2}-1\right)$, we have

$$
\begin{aligned}
& x^{-\alpha} \cdot H_{4}\left(\alpha, \sigma+a^{\prime}, \frac{\alpha+1}{2}, \frac{\alpha+1}{2}, \frac{x^{2}-1}{4 x^{2}}, \frac{y}{x}\right) \\
& =\sum_{k=0}^{\infty} \frac{(\alpha)_{k}\left(\sigma+a^{\prime}\right)_{k}}{((\alpha+1) / 2)_{k}((\alpha+1) / 2)_{k}} y^{k} \cdot P_{k}^{((\alpha-1) / 2,(\alpha-1) / 2)}(x) .
\end{aligned}
$$

On comparing the right-hand side of (3.10) with the left-hand side of formula [8, p. 271, (11)], we obtain (3.4).

(c) Setting

$$
A_{m n}=(\alpha)_{n-m}(\gamma)_{m}(\delta)_{m} / m ! n !
$$

in Theorem 2, we have, on simplification,

$$
\begin{aligned}
S(x, x y, \sigma) & =H_{2}\left(\alpha, \sigma+a^{\prime}, \gamma, \delta, \sigma+b^{\prime}, x y, x\right) \\
& =\sum_{n, m=0}^{\infty} \sum_{k=0}^{n} \frac{(\alpha)_{n-m}(\gamma)_{m}(\delta)_{m}\left(b^{\prime}-a^{\prime}\right)_{k}(-n)_{k}(x)^{m} \cdot(x y)^{n}}{m ! n ! k !\left(\sigma+b^{\prime}\right)_{k}} \\
& =\sum_{n, m, k=0}^{\infty} \frac{(\alpha)_{n+k-m}(\gamma)_{m}(\delta)_{m}\left(b^{\prime}-a^{\prime}\right)_{k}(x)^{m} \cdot(x y)^{n} \cdot(-x y)^{k}}{n ! k ! m !\left(\sigma+b^{\prime}\right)_{k}}
\end{aligned}
$$

on account of $[8$, p. $57,(2)]$.

In (3.11), summing the series over $n$, we obtain the result (3.5).

(d) In Theorem 2, taking

$$
A_{m n}=(\alpha)_{m+n}(\beta)_{m} / m ! n !(\gamma)_{m},
$$

we have, on simplification,

$$
\begin{aligned}
F_{2}(\alpha, \beta, \sigma & \left.+a^{\prime}, \gamma, \sigma+b^{\prime}, x, x y\right) \\
& =\sum_{m, n=0}^{\infty} \sum_{k=0}^{n} \frac{(\alpha)_{m+n}(\beta)_{m}\left(b^{\prime}-a^{\prime}\right)_{k}(-n)_{k}(x)^{m+n} \cdot(y)^{k}}{m ! n ! k !(\gamma)_{m}\left(\sigma+b^{\prime}\right)_{k}} \\
& =\sum_{m, n, k=0}^{\infty} \frac{(\alpha)_{m+n+k}(\beta)_{m}\left(b^{\prime}-a^{\prime}\right)_{k}(x)^{m} \cdot(x y)^{n} \cdot(-x y)^{k}}{m ! n ! k !(\gamma)_{m}\left(\sigma+b^{\prime}\right)_{k}}
\end{aligned}
$$

Inserting the factor $\left(\beta^{\prime}\right)_{n} /\left(\beta^{\prime}\right)_{n}$ on the right-hand side of (3.12), we obtain formula (3.6), which involves the Lauricella function $F_{A}[6$, p. 114].

(e) In Theorem 3, taking

$$
A_{m n}=(\beta)_{m}\left(\beta^{\prime}\right)_{n} / m ! n !
$$

we obtain 


$$
\begin{aligned}
F_{1}(\sigma & \left.+a, \beta, \beta^{\prime}, \sigma+b, x, x y\right) \\
& =\sum_{j=0}^{\infty} \frac{(b-a)_{j}(-x)^{j}}{j !(\sigma+b)_{i}} \frac{\partial^{j}}{\partial x^{j}}(1-x)^{-\beta} \cdot(1-x y)^{-\beta^{\prime}} \\
& =\sum_{i=0}^{\infty} \sum_{p=0}^{i} \frac{(b-a)_{i}(-j)_{p}(\beta)_{i-p}\left(\beta^{\prime}\right)_{p}(-1)^{p} \cdot(-x)^{i} \cdot(y)^{p} \cdot(1-x)^{-\beta-i+p}(1-x y)^{-\beta^{\prime}-p}}{j ! p !(\sigma+b)_{i}} \\
& =(1-x)^{-\beta}(1-x y)^{-\beta^{\prime}} \sum_{i, p=0}^{\infty} \frac{(b-a)_{i+p}(\beta)_{i}\left(\beta^{\prime}\right)_{p}}{p ! j !(\sigma+b)_{i+p}}\left(\frac{x}{x-1}\right)^{i} \cdot\left(\frac{x y}{x y-1}\right)^{p} \\
& =(1-x)^{-\beta} \cdot(1-x y)^{-\beta^{\prime}} \cdot F_{1}\left(b-a, \beta, \beta^{\prime}, \sigma+b, \frac{x}{x-1}, \frac{x y}{x y-1}\right) .
\end{aligned}
$$

Thus (3.7) is established. It is not difficult to reduce (3.7) to different forms [5, pp. 239, 240, (2)-(5)].

Special Cases. (i) In (3.3), substituting $\alpha=1$, we have the hypergeometric function $H_{4}$ as a generating function for the Legendre polynomial. Thus

$$
\begin{aligned}
x^{-1} \cdot H_{4}\left(1, \sigma+a^{\prime}, 1\right. & \left., \sigma+b^{\prime}, \frac{x^{2}-1}{4 x^{2}}, \frac{\left(x^{2}-1\right) y}{4 x^{2}}\right) \\
& =\sum_{k=0}^{\infty} \frac{\left(\sigma+a^{\prime}\right)_{k}}{\left(\sigma+b^{\prime}\right)_{k}}\left[\frac{\left(x^{2}-1\right) y}{4 x}\right]^{k} \cdot p_{k}(x) .
\end{aligned}
$$

Substituting $a^{\prime}=b^{\prime}$ and replacing $y$ by $4 x y /\left(x^{2}-1\right)$ in (3.13), we obtain, on simplification, a known generating function $[8$, p. 156 , (1)] for the Legendre polynomial.

(ii) In (3.5), replacing $y$ by $y / x, x$ by $x / \delta$ and letting $\delta$ tend to infinity, we obtain

$$
\begin{aligned}
H_{2}(\alpha, \sigma & \left.+a^{\prime}, \gamma, \sigma+b^{\prime}, y, x\right) \\
& =(1-y)^{-\alpha} \cdot H_{2}\left(\alpha, b^{\prime}-a^{\prime}, \gamma, \sigma+b^{\prime}, \frac{y}{y-1}, x(1-y)\right),
\end{aligned}
$$

where $H_{2}$ is the confluent hypergeometric function [5, p. 226, (30)].

Again in (3.14), replacing $x$ by $x / \gamma$ and letting $\gamma$ tend to infinity, we have the transformation formula

$$
\begin{aligned}
H_{3}\left(\alpha, \sigma+a^{\prime}, \sigma+b^{\prime}, y, x\right) \\
\quad=(1-y)^{-\alpha} \cdot H_{2}\left(\alpha, b^{\prime}-a^{\prime}, \sigma+b^{\prime}, \frac{y}{y-1}, x(1-y)\right)
\end{aligned}
$$

where $H_{3}$ is the hypergeometric function [5, p. 226, (31)].

(iii) In (3.12), summing the series over $n$, we obtain a known transformation formula [5, p. 240, (7)].

(iv) Again, in (3.12), summing the series over $m$, employing formula [5, p. 105, (3)] and expressing the hypergeometric function ${ }_{2} F_{1}$ again in series form, we have

$$
\begin{aligned}
& F_{2}\left(\alpha, \beta, \sigma+a^{\prime}, \gamma, \sigma+b^{\prime}, x, x y\right)=(1-x)^{-\alpha} \\
& \quad \cdot \sum_{n, k, m=0}^{\infty} \frac{(\alpha)_{n+k+m}\left(b^{\prime}-a\right)_{k}(\gamma-\beta)_{m}}{n ! k ! m !\left(\sigma+b^{\prime}\right)_{k}(\gamma)_{m}}\left(\frac{x y}{1-x}\right)^{n} \cdot\left(\frac{x y}{x-1}\right)^{k} \cdot\left(\frac{x}{x-1}\right)^{m} .
\end{aligned}
$$


In (3.16), summing the series over $n$, we obtain a known transformation formula $[5$, p. $240,(8)]$.

Similarly, (3.16) can also lead to [5, p. 240, (6)].

4. In this section, making use of Theorem 1 of Section 2, we establish the asymptotic confluent expansion for the Kampé de Fériet function.

We prove the formula

$$
\begin{aligned}
& F_{\nu+1, \mu, \mu+1}^{\lambda+1, \mu+1}\left[\begin{array}{c|l}
x & a_{\lambda}, \sigma+a: b_{\mu} ; b_{\mu}^{\prime}, \sigma+a^{\prime} \\
x y & a_{\nu}^{\prime}, \sigma+b: c_{\rho} ; c_{\rho}^{\prime}, \sigma+b^{\prime}
\end{array}\right] \\
& \sim F_{\nu, \rho}^{\lambda, \mu}\left[\begin{array}{c|c}
x & a_{\lambda}: b_{\mu} ; b_{\mu}^{\prime} \\
x y & a_{\nu}^{\prime}: c_{\rho} ; c_{\rho}^{\prime}
\end{array}\right]+\frac{1}{(\sigma+b)} A_{1,0}(x, x y)+\frac{1}{\left(\sigma+b^{\prime}\right)} A_{0,1}(x, x y) \\
& +\frac{1}{(\sigma+b)\left(\sigma+b^{\prime}\right)} A_{1,1}(x, x y)+\frac{1}{(\sigma+b)_{2}} A_{2,0}(x, x y) \\
& +\frac{1}{\left(\sigma+b^{\prime}\right)_{2}} A_{0,2}(x, x y) \\
& +\frac{1}{\left(\sigma+b^{\prime}\right)_{3}} A_{0,3}(x, x y)+\frac{1}{(\sigma+b)_{3}} A_{3,0}(x, x y) \\
& +\frac{1}{(\sigma+b)_{2}\left(\sigma+b^{\prime}\right)} A_{2,1}(x, x y)+\frac{1}{(\sigma+b)\left(\sigma+b^{\prime}\right)_{2}} A_{1,2}(x, x y)+\cdots, \\
& \sigma \rightarrow \infty, \lambda+\mu \leqq \nu+\rho+1 \text {, and } \\
& A_{r, s}(x, x y)=\frac{(-x)^{r}(-y)^{s}(b-a)_{r}\left(b^{\prime}-a^{\prime}\right)_{s}}{r ! s !} \frac{\partial^{r+s}}{\partial x^{r} \cdot \partial y^{s}} F_{\nu, \rho}^{\lambda, \mu}\left[\begin{array}{l|l}
x & a_{\lambda}: b_{\mu} ; b_{\mu}^{\prime} \\
x y & a_{\nu}^{\prime}: c_{\rho} ; c_{\rho}^{\prime}
\end{array}\right] .
\end{aligned}
$$

Proof. In Theorem 1, setting $S(x, x y, \sigma)$ equal to the Kampé de Fériet function given on the left-hand side of (4.1), and making use of (2.4) and (2.8), we arrive at (4.1).

By reducing the Kampé de Fériet function to Appell functions, many special cases of (4.1) can be obtained.

\section{Pratap College}

Amalner (Jalgaon)

Maharashtra, India

1. V. L. DeshPande, "Theorems involving confluent asymptotic expansions of functions of two variables," An. Univ. Timisoara Romania, v. 8, 1970, fasc. 2, pp. 143-151.

2. V. L. DESHPANDE, "Theorems on asymptotic confluent expansions for functions of two variables," J. Natur. Sci. and Math., v. 11, 1971, no. 1.

3. V. L. DESHPANDE \& V. M. BHISE, "On asymptotic confluent expansions for functions of two variables," Nederl. Akad. Wetensch. Proc. Ser. A, v. $75=$ Indag. Math., v. 34, 1972, no. 2.

4. JERry L. Fields, "Confluent expansions," Math. Comp., v. 21, 1967, pp. 189-197. MR 37 \#49.

5. A. Erdélyi, Higher Transcendental Functions. Vol. I. The Hypergeometric Function, Legendre Functions, McGraw-Hill, New York, 1953. MR 15, 419.

6. P. APPELL \& J. KAMPÉ DE FÉRIET, Fonctions hypergéométriques et hypersphériques polynômes d'Hermite, Gauthier-Villars, Paris, 1926.

7. F. G. TRICOMI \& A. ERDÉLYI, "The asymptotic expansion of a ratio of gamma functions," Pacific J. Math., v. 1, 1951, pp. 133-142. MR 13, 343.

8. E. D. RAINviLLE, Special Functions, Macmillan, New York, 1960. MR 21 \#6447. 